This item was submitted to Loughborough's Research Repository by the author.

Items in Figshare are protected by copyright, with all rights reserved, unless otherwise indicated.

\title{
The use of holographic optics for heat flow control in wire-based laser
}

\section{cladding}

PLEASE CITE THE PUBLISHED VERSION

PUBLISHER

ICALEO 2014 Proceedings (c) Laser Institute of America

VERSION

AM (Accepted Manuscript)

PUBLISHER STATEMENT

All rights reserved

LICENCE

All Rights Reserved

REPOSITORY RECORD

Goffin, Nick, Shuai Hou, John Tyrer, and Rebecca Higginson. 2019. "The Use of Holographic Optics for Heat Flow Control in Wire-based Laser Cladding”. figshare. https://hdl.handle.net/2134/16837. 


\title{
THE USE OF HOLOGRAPHIC OPTICS FOR HEAT FLOW CONTROL IN WIRE-BASED LASER CLADDING
}

Paper Number (\#501)

\author{
N.J. Goffin ${ }^{\text {a }}$, S. Hou ${ }^{\text {a }}$, J.R. Tyrer ${ }^{\text {a }}$, R.L. Higginson ${ }^{b}$ \\ ${ }^{a}$ Wolfson School of Mechanical and Manufacturing Engineering, Loughborough University, \\ Loughborough, United Kingdom, LE11 3TU \\ ${ }^{\mathrm{b}}$ Department of Materials, Loughborough University, Loughborough, United Kingdom, LE11 3TU
}

\begin{abstract}
Laser cladding with wire utilises a focussing lens to melt the surface of the substrate, into which the wire is fed to build up a clad track on the surface. Process reliablity issues in practice include; clad tracks with high levels of dilution, surface cracking and other defects.

Key to this is wire reflectivity calculations. Here using Fresnel equations that relate angle of incidence to heat absorption, we are able to show a direct correlation between the applied heat profile of the laser beam and the absorption profile of the wire surface; this has been modelled using COMSOL multiphysics conduction simulations which showed that the heat profile of the applied laser beam has a direct effect on the size and shape of the resulting melt pool.
\end{abstract}

Using computer generated Holographic Optical Elements (HOE), a novel form of optic that alters the heat profile of the laser beam to a user-specified 3d profile, a conventional $1.25 \mathrm{~mm}$ diameter Gaussian beam shape and a $1.25 \mathrm{~mm}$ square uniform 'pedestal' HOE-derived beam shape were tested and compared, using a $1 \mathrm{~mm}$ diameter AISI 316 stainless steel wire on a $0.8 \mathrm{~mm}$ mild steel substrate.

These results were also compared to an enlarged 3.5 $\mathrm{mm}$ diameter Gaussian beam, in order to evaluate different methods of altering the heat distribution applied to the wire. The HOE generated beam gave superior results, due to its shorter thermal cycle, which reduced the amount of heat going into the clad track and resulted in lower dilution.

\section{Introduction}

Laser cladding with wire can be considered to be an addition modification of the conduction-limited laser welding process, where the filler wire is a different material to the substrate and is laid on top of it; instead of being used to bulk out the weld pool. When it comes to welding, lasers have certain advantages over other heat sources with respect to the heat density that can be applied, the processing speed and the ability to carry out welding in remote locations: through windows and inside complex three-dimensional components [1].

Clad materials are most commonly fed into the melt pool in one of two ways; either in the form of powder or wire. In industry, the majority of laser cladding processes are powder-based. Powder is either preplaced onto the work-piece or blown into the melt pool using a gas jet, either co-axially or using an offset feeding nozzle arrangement. A comparative study of these two methods was conducted by Syed et al. [2], who found distinct advantages and disadvantages to both, with the main advantages of wire relating to its feed rate and deposition efficiency and the advantages of powder relating to the greater range of possible feed angles.

Other advantages of wire include its suitability for automatic production, since it is easier to regulate the feeding of wire than the blowing of powder and the ease of adapting a wire feeder to awkward cladding positions, such as the inside of tubes [3], or other directions, such as overhead cladding. Wire feeding's main disadvantage is the sensitivity of the process to changes in processing parameters [4], and a tendency for wire-created clad tracks to have high incidence of porosity, high dilution and surface cracking [5]. These disadvantages mean that wire feeding methods are not widely used in industry.

Computer generated Holographic Optical Elements provide the ability to escape the circular beam profile and redistribute the beam energy into a user defined distribution[6], a variation of the HOE known as a kinoform allows much higher diffraction efficiency elements to be produced, up to $95 \%$ of the incident beam converted into the shape and power distribution desired. They have been found to be effective in metal processing, where they are used to selectively alter the thermal cycle that the processing zone undergoes. Since the thermal cycle directly affects the resulting 
metallurgy [1], this allows the microstructure of the processing zone to be controlled.

In laser welding, they were used by Kell et al. [7] to control the shape and microstructure of the melt pool in mild and stainless steel. This was followed up by Gibson et al. [8], who investigated their use in powderbed laser cladding; firstly with stainless steel and then with Stellite and Inconel. This paper discusses their use in control of melting behaviour for laser cladding with wire as a source material.

\section{Experimental Procedure}

Experiments were carried out using a $1.2 \mathrm{~kW}$ Coherent Everlase $\mathrm{S}_{4} \mathrm{CO}_{2}$ laser set at a wavelength of $10.6 \mu \mathrm{m}$, run in $\mathrm{CW}$ mode.

AISI 316 stainless steel wire was used as the clad material and $0.8 \mathrm{~mm}$ mild steel sheet was used as the substrate material. Alloy compositions are given in Table 1 and Table 2.

Table 1: Alloy compositions of experimental materials

\begin{tabular}{|l|c|c|c|c|c|c|}
\hline $\begin{array}{l}\text { Element } \\
\text { (mild } \\
\text { steel) }\end{array}$ & $\mathrm{Mn}$ & $\mathrm{C}$ & $\mathrm{Cr}$ & $\mathrm{Cu}$ & $\mathrm{S}$ & $\mathrm{Fe}$ \\
\hline $\begin{array}{l}\% \\
\text { Weight }\end{array}$ & 0.79 & 0.15 & 0.08 & 0.05 & 0.02 & balance \\
\hline
\end{tabular}

Table 2: Alloy composition of AISI 316 stainless steel

\begin{tabular}{|l|c|c|c|c|c|c|}
\hline $\begin{array}{l}\text { Element } \\
\text { (stainless } \\
\text { steel) }\end{array}$ & $\mathrm{Cr}$ & $\mathrm{Ni}$ & $\mathrm{Mo}$ & $\mathrm{Mn}$ & $\mathrm{Si}$ & $\mathrm{Fe}$ \\
\hline $\begin{array}{l}\% \\
\text { Weight }\end{array}$ & 18.26 & 12.09 & 2.53 & 1.61 & 0.42 & balance \\
\hline
\end{tabular}

The substrate was laser cut into coupons, each $50 \times 15$ $\mathrm{mm}$, for laser cladding; one clad track per coupon. Instead of feeding the wire in with a feeding mechanism, it was clamped down onto the substrate in advance to avoid the thermal effects associated with wire feeding [9], so as to match the experimental conditions with the simulated conditions as closely as possible.

The work piece had a delay of 4 seconds between the initiation of the laser beam and commencing the traverse. This was to allow a melt pool to initiate. The work piece then traversed for $35 \mathrm{~mm}$ at a rate of 1.6 $\mathrm{mm} / \mathrm{s}$.

A Gaussian beam with a $1 / \mathrm{e}^{2}$ diameter of $1.25 \mathrm{~mm}$ was test against a $1.25 \mathrm{~mm}$ square flat 'pedestal' beam profile created with a HOE, creating a similar cladding beam width but different power density distribution. The HOE beam was then tested against a $1 / \mathrm{e}^{2}$ diameter $3.5 \mathrm{~mm}$ Gaussian beam which was created by defocussing the laser using a conventional focussing lens. The beam profiles are shown in Figure 1.
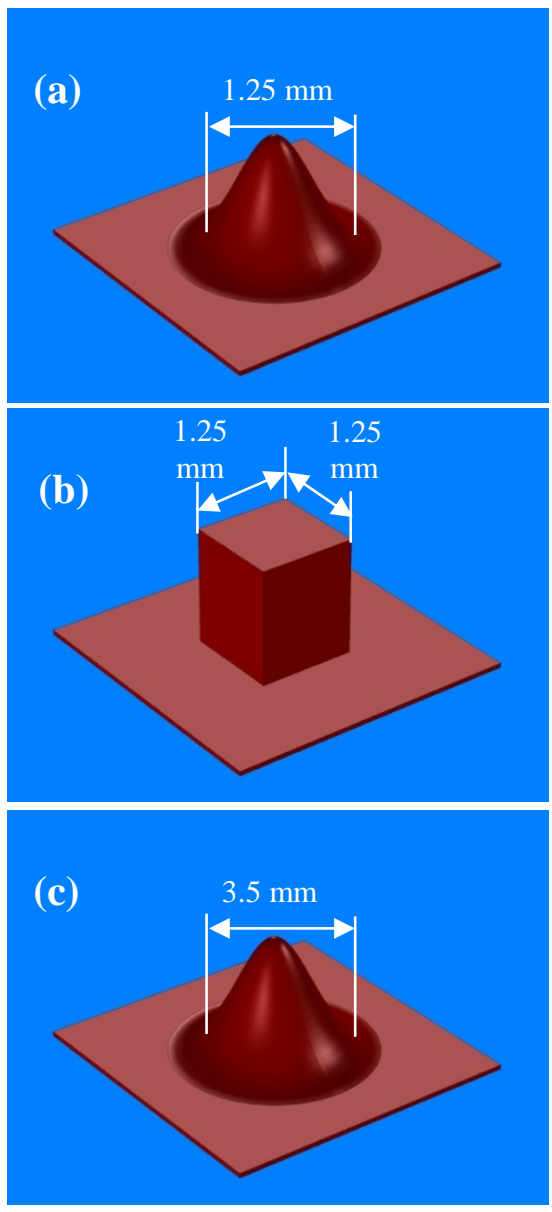

Figure 1: Laser beam heat profiles (a) $1.25 \mathrm{~mm}$ Gaussian beam (b) 1.25 mm pedestal HOE beam (c) 3.5 mm Gaussian beam

Clad samples were sectioned and mounted in Bakelite. They were then ground and polished to $1 \mu \mathrm{m}$ and etched with Kalling's \#2 and Schaftmeister's reagents.

Optical micrographs were created using a Nikon Optiphot optical microscope mounted with a QImaging Micropublisher 3.3 digital camera. Image scaling and measurement were conducted using Image Pro Premier 9.1 software. 


\section{Surface Reflectivity Calculations}

Because the wire has a circular cross-section, the surface that interacts with the laser beam is curved, which means that the angle of incidence changes across its width, increasing from $0^{\circ}$ at the centre to $90^{\circ}$ at a tangent to the outer edge. A plot of incidence angle vs. this transverse distance from the centre is shown in Figure 8. Since both the wire and laser beam are symmetric, only one half was plotted.

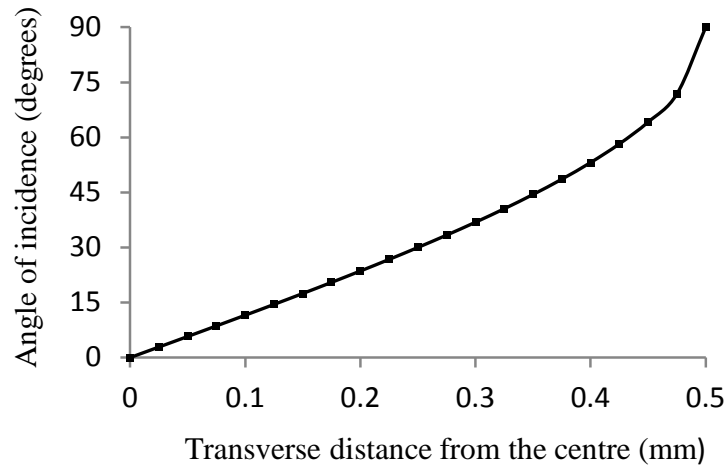

Figure 2: Graph cross-referencing transverse distance across the wire with laser beam incidence angle

Figure 2 reveals a mostly linear increase in incidence angle across the transverse width. This increase in incidence angle affects the reflectivity of the wire across its width, which then affects heat absorption and how the wire melts onto the substrate.

Metals have a complex index of refraction, in the form:

$$
m=n-j k
$$

Where $m$ is the complex index of refraction, $n$ is the refractive index and $k$ is the damping constant [10]. These values are dependent on both the wavelength of the light, and the material being used. Boyden and Zhang determined the values for a $10.6 \mu \mathrm{m} \mathrm{CO}_{2}$ laser on AISI 304 stainless steel [11]:

$$
\begin{array}{ll}
\text { n-value } & \text { k-value } \\
20.4 & 21.5
\end{array}
$$

Incidence angles were calculated at points across the width of the wire. These angles were then used in Fresnel equations to calculate the reflectivity at each point. For metals, these equations simplify to [10]:

$\begin{aligned} & \text { Parallel- } \\ & \text { polarised }\end{aligned} \quad \rho_{\|}=\frac{(\mathrm{n} \cos \theta-1)^{2}+(\mathrm{k} \cos \theta)^{2}}{(\mathrm{n} \cos \theta+1)^{2}+(\mathrm{k} \cos \theta)^{2}}$

light: $\begin{aligned} & \text { Perpendicular- } \\ & \text { polarised light: }\end{aligned} \quad \rho_{\perp}=\frac{(\mathrm{n}-\cos \theta)^{2}+\mathrm{k}^{2}}{(\mathrm{n}+\cos \theta)^{2}+\mathrm{k}^{2}}$

Where $\rho$ is reflectivity, $\theta$ is incidence angle and $n$ and $k$ are already defined.

For a circularly polarised laser beam, the mean is found of Equations 2 and 3:

$$
\rho=\frac{\rho_{\|}+\rho_{\perp}}{2}
$$

Where $\rho$ is the mean reflectivity. From this, the reflectivity profile of the wire from the centre to the outer edge was plotted. The plot is shown in Figure 9.

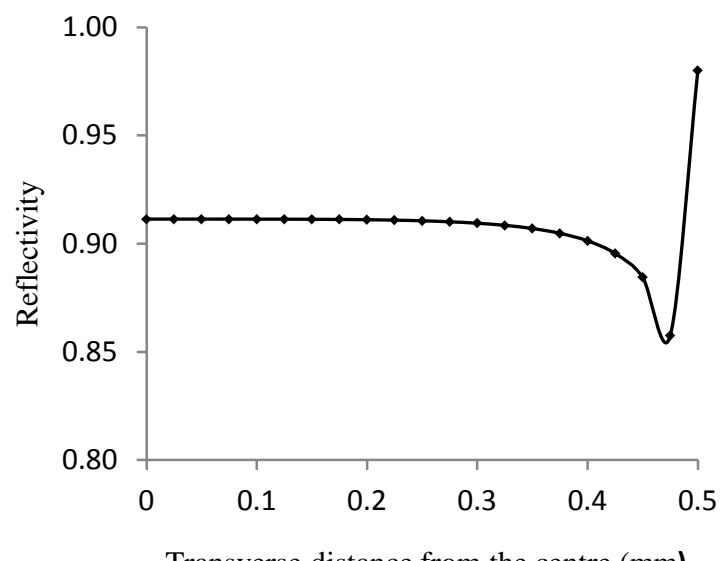

Figure 3: Graph of reflectivity vs. transverse distance from the centreline

Figure 3 reveals a flat reflectivity profile, with a large decrease in reflectivity between 0.4 and $0.5 \mathrm{~mm}$ from the centre. Using Figure 8, this translates to incidence angles between $45-75^{\circ}$.

Both Gaussian [12] and flat input profiles were applied to this reflectivity curve with a nominal laser power of $100 \mathrm{~W}$, giving a plot of absorbed irradiance on a circular wire for those beam profiles. Both beam widths were defined at $1.25 \mathrm{~mm}$, slightly larger than the wire diameter, and the Gaussian beam width used the $1 / \mathrm{e}^{2}$ definition of diameter. Figure 4 shows the generated plots. 


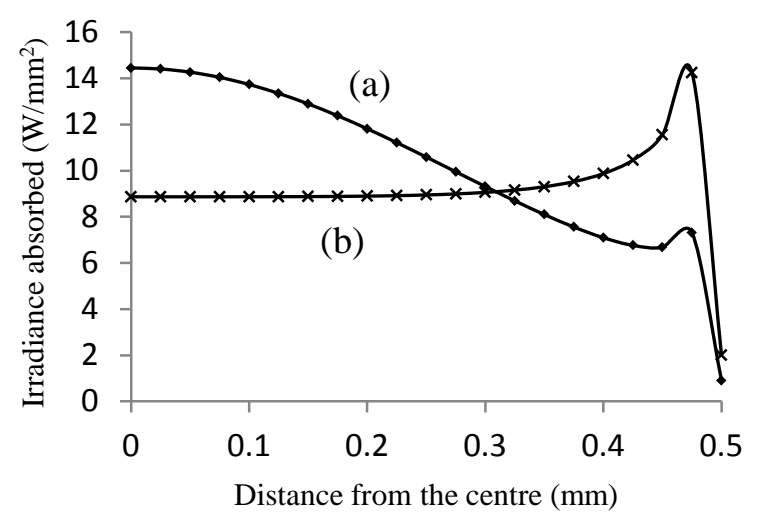

Figure 4: Absorbed irradiance distributions for (a) Gaussian and (b) flat laser beam profiles

The Gaussian irradiance distribution shows an increased level of absorption in the centre of the wire, approximately double the amount at the edge. The flat irradiance distribution increases towards the edge, corresponding to the increase in wire absorption. Both have absorption "spikes" at the outer edges of the wire, where the $45-75^{\circ}$ incidence angle range is present, and this spike is more pronounced on the flat beam than the Gaussian one.

The shape of the wire has a greater effect on the flat beam than the Gaussian beam, causing a concentration of energy at the outer edge that is much less present in the Gaussian beam, whose absorption profile is broadly the same as the raw beam profile.

Apart from using HOE's, another possible method of increasing the energy present at the edge of the wire is to increase the size of the laser beam. A schematic of this method is shown in Figure 5.

(a) $1.25 \mathrm{~mm}$ Gaussian beam

(b) Larger Gaussian beam

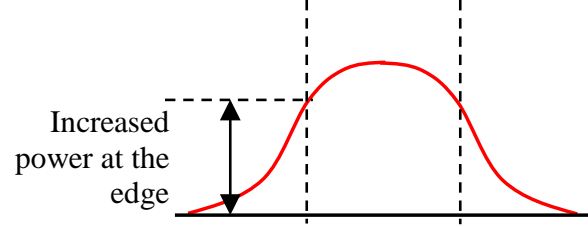

Figure 5: Schematic of effective power distribution when increasing the size of the applied Gaussian beam showing (a) Original $1.25 \mathrm{~mm}$ Gaussian beam and (b) larger beam
Applying this larger beam through the surface reflectivity profile of the wire, gives an absorption profile that is very similar to that of a pedestal HOE beam. This is shown with a $3.5 \mathrm{~mm}$ beam in Figure 6 .

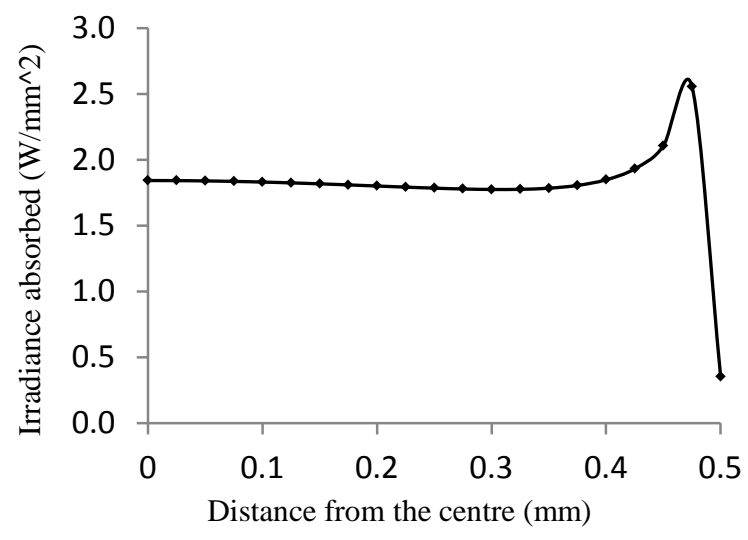

Figure 6: Laser beam absorption profile for $3.5 \mathrm{~mm}$ diameter Gaussian beam on $1 \mathrm{~mm}$ diameter wire

\section{Surface Reflectivity for Experimental Beams}

In experiments, three clad tracks were created for each beam type: A track that was under-penetrated, a track that was over-penetrated and a track that was between those two. The laser power output required for these clad tracks was put through the reflectivity calculations and the following irradiance data types were obtained (schematic in Figure 7):

- $\quad$ Absorbed Irradiance in the Centre (AIC). This was calculated by inputting applied power to the absorption calculations and finding the absorbed irradiance in the centre of the beam/wire.

- $\quad$ Absorbed Irradiance at the outer Edge (AIE). This was calculated the same way as the AIC but with the absorbed irradiance at the outer edge, in the middle of the edge absorption peak.

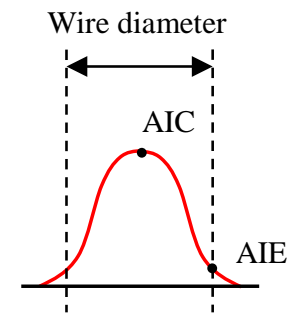

Figure 7: Schematic of irradiance positions 
Absorbed irradiance data for AIC and AIE are given in Table 3.

Table 3: Irradiance data for experimental beam types

\begin{tabular}{|c|c|c|c|}
\cline { 2 - 4 } \multicolumn{1}{c|}{} & $\begin{array}{c}\text { Test } \\
\text { number }\end{array}$ & $\begin{array}{c}\text { AIC } \\
\left(\mathrm{W} / \mathrm{mm}^{2}\right)\end{array}$ & $\begin{array}{c}\text { AIE } \\
\left(\mathrm{W} / \mathrm{mm}^{2}\right)\end{array}$ \\
\hline \multirow{3}{*}{$\begin{array}{c}1.25 \mathrm{~mm} \\
\text { Gaussian beam }\end{array}$} & 1 & 85 & 43 \\
\cline { 2 - 4 } & 3 & 89 & 45 \\
\hline \multirow{3}{*}{$\begin{array}{c}1.25 \text { mm } \\
\text { pedestal beam }\end{array}$} & 5 & 15 & 24 \\
\cline { 2 - 4 } & 6 & 19 & 30 \\
\hline \multirow{3}{*}{$\begin{array}{c}3.5 \text { mm } \\
\text { Gaussian beam }\end{array}$} & 8 & 6 & 9 \\
\cline { 2 - 4 } & 9 & 8 & 12 \\
\cline { 2 - 4 } & 7 & 7 & 10 \\
\hline
\end{tabular}

For a $1.25 \mathrm{~mm}$ Gaussian beam, the absorbed irradiance at the centre is twice that of the edge. When a pedestal beam is applied, absorption at the edge is increased by $50 \%$ over that in centre. This ratio also holds true for the $3.5 \mathrm{~mm}$ Gaussian beam, with approximately $50 \%$ more absorption at the edge than the centre.

\section{Thermal Conduction Simulations}

Thermal conduction simulations were carried out using COMSOL Multiphysics. Figure 8 shows heat conduction simulations for the $1 / \mathrm{e}^{2} 1.25 \mathrm{~mm}$ Gaussian, $1.25 \mathrm{~mm}$ pedestal and $1 / \mathrm{e}^{2} 3.5 \mathrm{~mm}$ Gaussian heat profiles; all at a $300 \mathrm{~W}$ input power. The white arrows represent heat flux, the direction and density of heat flow and the thick white line represents the melt boundary; the point which the melt pool reaches before solidifying.

The simulations reveal the following information:

- For the $1.25 \mathrm{~mm}$ Gaussian beam, the wire temperature reaches $3500 \mathrm{~K}$ but the heat distribution means that this is mainly applied centrally, resulting in a small predicted melt pool, despite the high wire temperature. The combination of a high wire temperature and small melt pool suggests that use of a 1.25 $\mathrm{mm}$ Gaussian beam will not produce a successful clad track, since at $300 \mathrm{~W}$, the wire temperature is triple the melting point of stainless steel, without creating a melt pool of any significant width.

- The pedestal beam places more heat at the edges of the wire, which raises it to a similar temperature to the $1.25 \mathrm{~mm}$ Gaussian beam, but with a different distribution. This results in more heat being conducted into the substrate and the formation of a larger melt pool.

- $\quad$ At $300 \mathrm{~W}$, the much larger area of the $3.5 \mathrm{~mm}$ Gaussian beam reduces the applied power density, therefore reducing the temperature. The temperature is still high enough to melt the wire, but does not conduct to the substrate sufficiently to form a significant melt pool. This suggests that a higher power than $300 \mathrm{~W}$ would be required to create a melt pool.

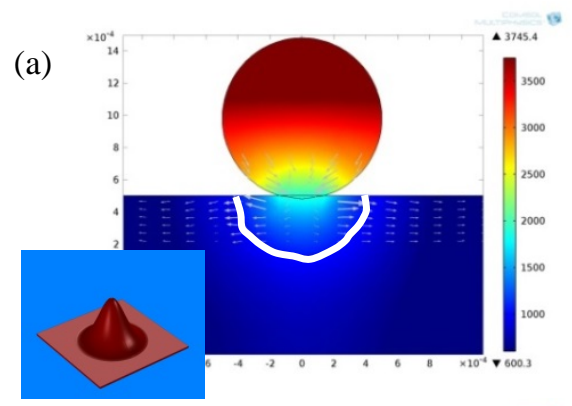

(b)
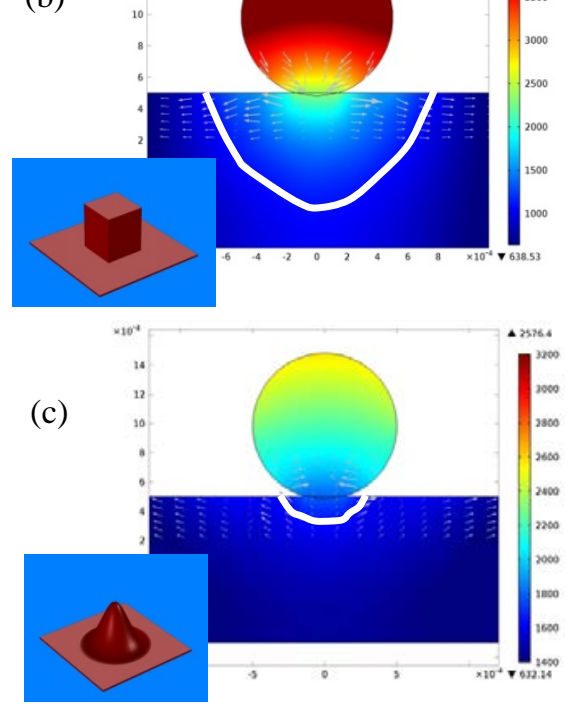

Figure 8: COMSOL simulations of diameter $1 \mathrm{~mm}$ wire illuminated by (a) $1.25 \mathrm{~mm}$ Gaussian beam (b) $1.25 \mathrm{~mm}$ pedestal beam (c) 3.5 mm Gaussian beam at $300 \mathrm{~W}$ power. 
Figure 9 shows the result when the wire is processed with a $3.5 \mathrm{~mm}$ Gaussian beam with power increased to $400 \mathrm{~W}$.

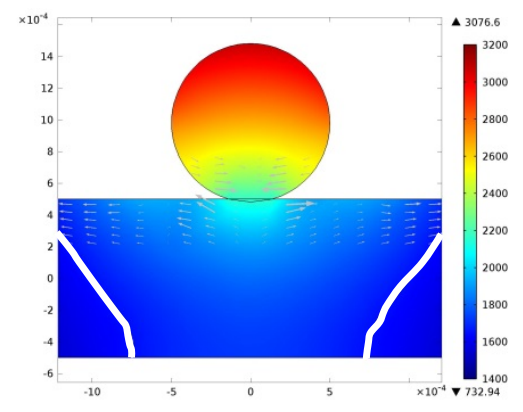

Figure 9: COMSOL simulation of $3.5 \mathrm{~mm}$ gaussian beam at $400 \mathrm{~W}$ power

The wire temperature now approaches the $3000+\mathrm{K}$ of the smaller beam sizes. The melt pool is now also much wider and has fully penetrated to the opposite side of the substrate. The simulation therefore predicts melt pool formation for the $3.5 \mathrm{~mm}$ Gaussian beam.

\section{Results and discussion}

Figure 10 shows micrographs of clad tracks created with the $1.25 \mathrm{~mm}$ Gaussian beam at a series of power densities. Notably, there is no melt pool formation seen. The wire melts and fuses with the substrate, without forming a melt pool. Once the power density is high enough, full fusion with the substrate occurs.

This shows what happens when the absorbed irradiance in the centre is much higher than that at the outer edge. The inner part melts and fuses while the outer part is not heated for enough time, or with a high enough irradiance, to flow. This is described in further detail by Figure 12 .

Once the applied power density is high enough for the AIE to melt the wire, the AIC generates an excess of heat, which causes the wire to mix and fully fuse with the substrate as soon as the outer edges melt and flow.

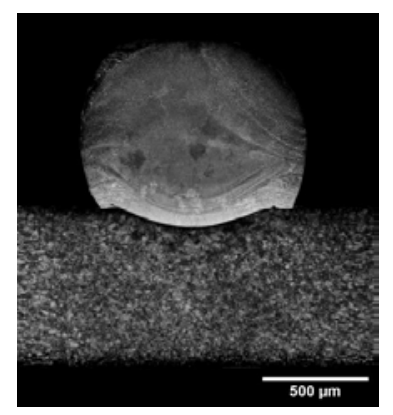

Test 1

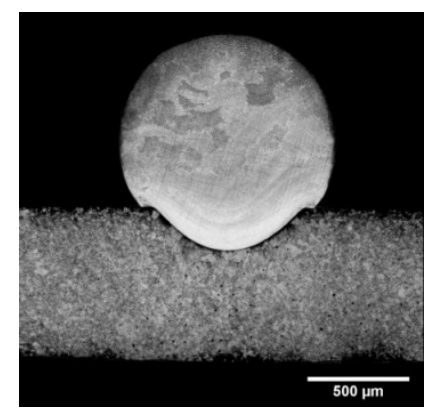

Test 2

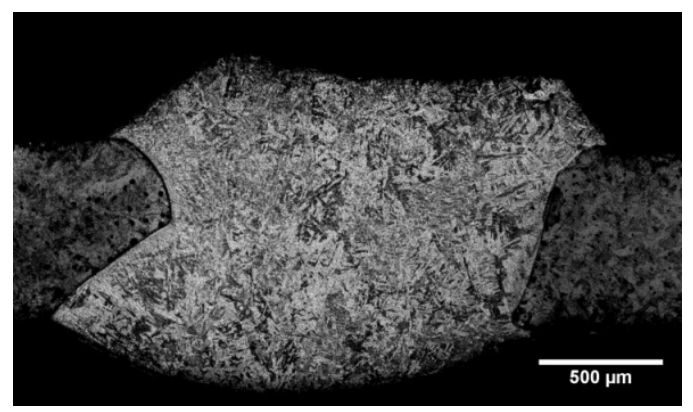

Test 3

Figure 10: Bright field optical micrographs showing melting behaviour of $1 \mathrm{~mm}$ diameter round wire illuminated with a $1.25 \mathrm{~mm}$ Gaussian beam, etched with Kalling's \#2 reagent

This contrasts with the pedestal; micrographs of which are shown in Figure 11. When additional heat is placed at the edges of the wire, it forms a melt pool much more readily and does not over-penetrate.

There is still a large amount of marangoni flow present in the melt pool however. The evidence for this flow is the small dimple present in middle of the clad tracks' top surfaces and the fact that there is still significant penetration.

Because the use of a pedestal HOE increases the AOE by approximately $50 \%$ compared to the AOC, the temperature at the edges is raised sufficiently to allow melting, without the centre becoming too hot. This 
allows a melt pool to form without the excess mixing that is present in the $1.25 \mathrm{~mm}$ Gaussian tests.

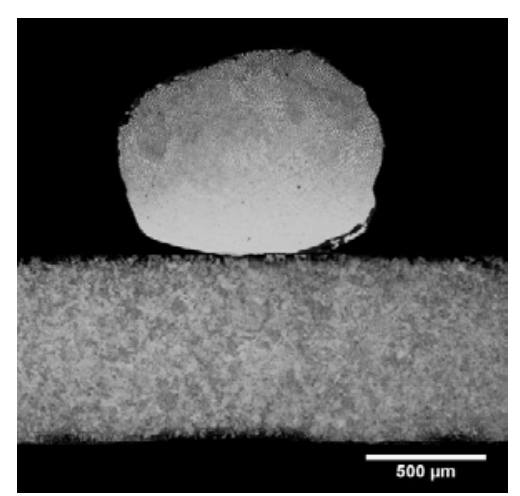

Test 4

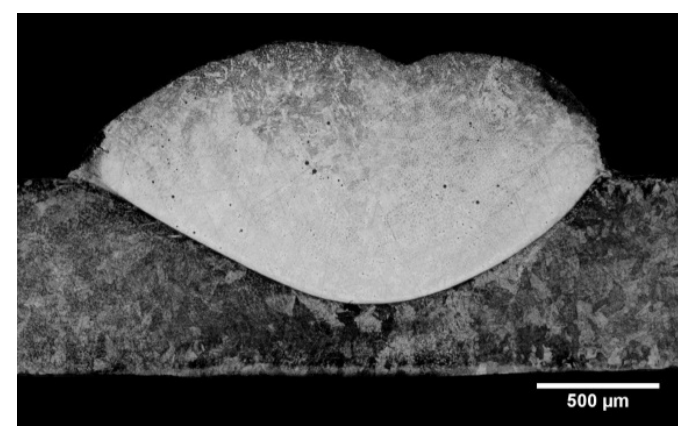

Test 5

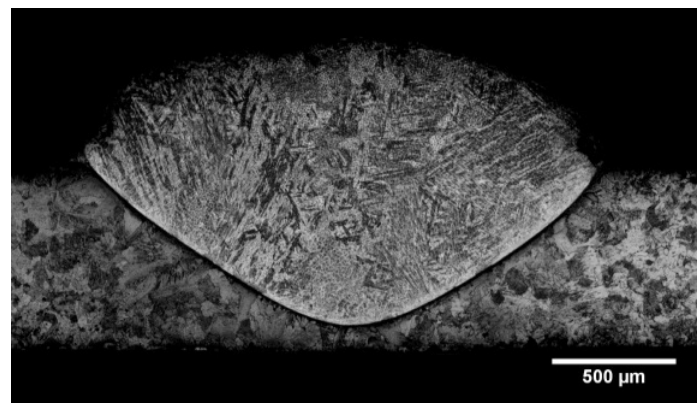

Test 6

Figure 11: Bright field optical micrographs showing melting behaviour of $1 \mathrm{~mm}$ diameter round wire illuminated with a $1.25 \mathrm{~mm}$ square pedestal beam, etched with Kalling's \#2 reagent

One other factor to take into account is the thermal cycle along the length of the clad, as well as across the width.

With a $1.25 \mathrm{~mm}$ Gaussian beam, the centre of the wire not only receives the highest concentration of energy, but also, because the beam is circular, receives it $0.8 \mathrm{~s}$; which is the time taken for the diameter of the beam to traverse. The edge irradiance is $50 \%$ less than the centre and it is illuminated for $0.4 \mathrm{~s}$, half the amount of time. The pedestal HOE beam, by contrast, not only has an additional 50\% irradiance at the edge, but its square shape means that all parts of the wire are illuminated for $0.8 \mathrm{~s}$. This difference is shown in Figure 12.

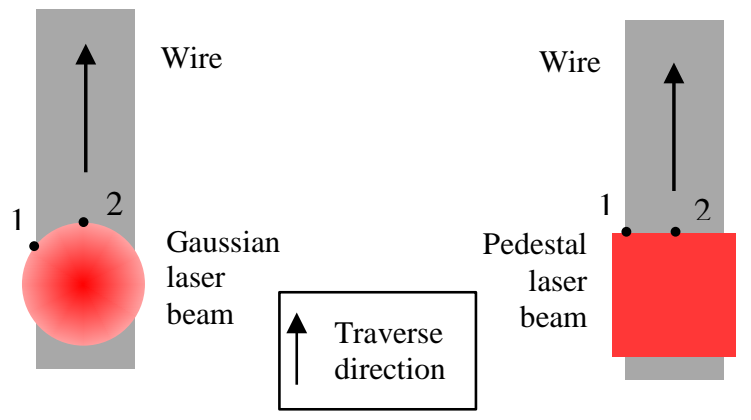

Figure 12: Schematic of Gaussian vs. pedestal beam heating times

This explains why the $1.25 \mathrm{~mm}$ Gaussian clad tracks combined over-penetration at the centre with poor flow at the edges whereas the $1.25 \mathrm{~mm}$ HOE clad tracks displayed much more even melting.

Experiments were then carried out with a $3.5 \mathrm{~mm}$ Gaussian beam. Tracks created with this beam shape are shown in Figure 13. 


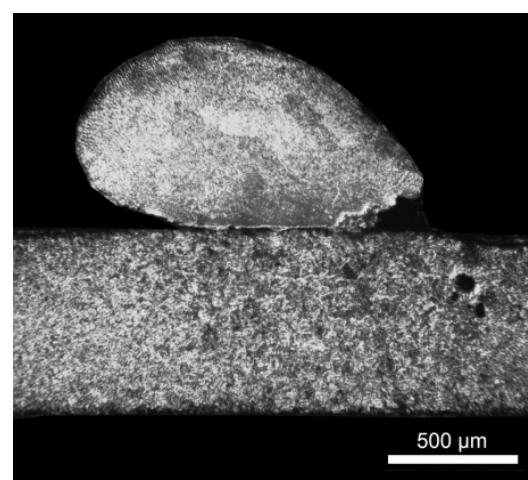

Test 7

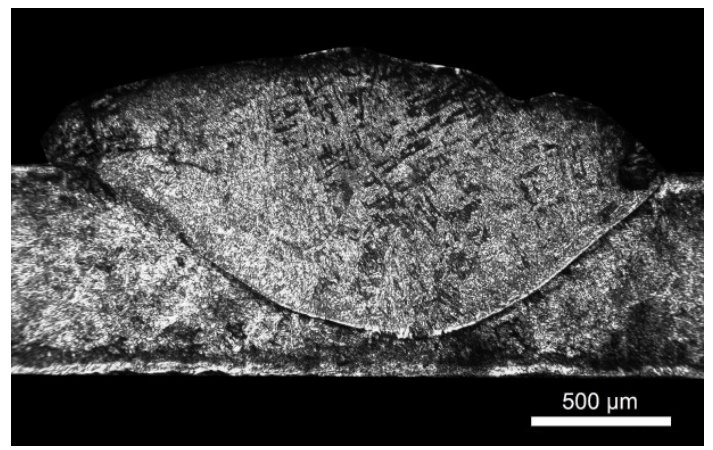

Test 8

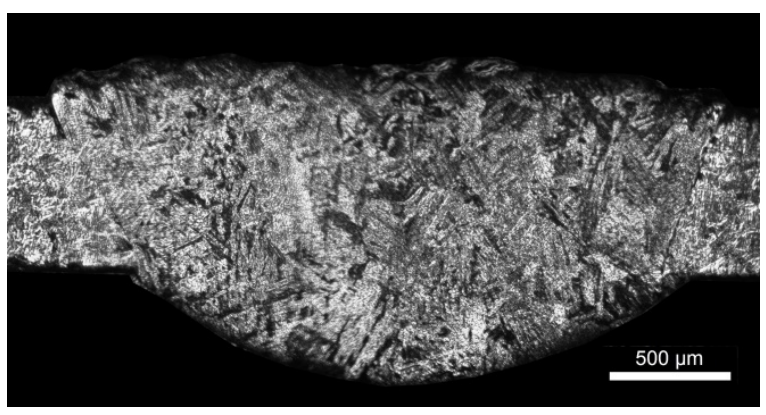

Test 9

Figure 13: Bright field optical micrographs showing melting behaviour of $1 \mathrm{~mm}$ diameter round wire illuminated with a $3.5 \mathrm{~mm}$ Gaussian beam, etched with Kalling's \#2 reagent

Reflectivity calculations showed that the laser-material interaction of a $3.5 \mathrm{~mm}$ Gaussian beam with a $1 \mathrm{~mm}$ diameter wire is very similar in its profile to that of a $1.25 \mathrm{~mm}$ pedestal HOE.

This is reflected in the experimental results, which show that this beam type is also capable of forming a melt pool. However, clad tracks created with this beam type display a greater tendency to mix with the substrate than those created with a pedestal HOE. This is probably due to the differences in heating time.

\section{Comparison Between Low Penetration $3.5 \mathrm{~mm}$ Gaussian Clad Track and Pedestal HOE Clad Track}

Two clad tracks were created for the different beam shapes at intermediate power settings designed to give melt pools with minimal penetration. These are shown in Figure 14.

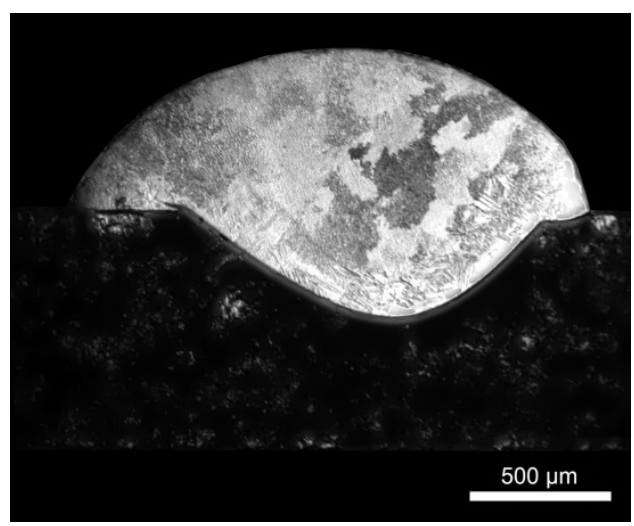

(a)

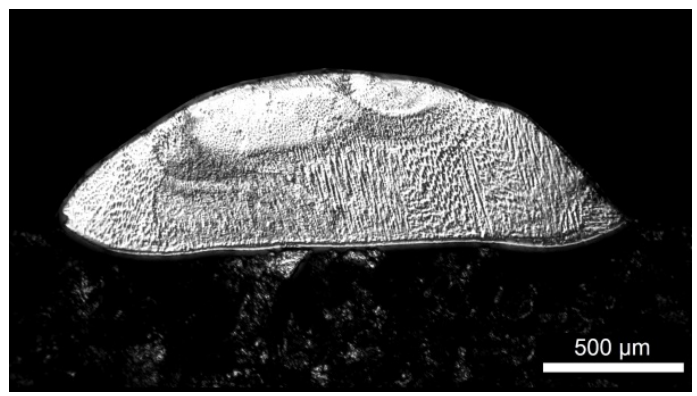

(b)

Figure 14: Optical micrographs showing (a) clad track created with a $3.5 \mathrm{~mm}$ Gaussian beam and (b) a clad track created with a $1.25 \mathrm{~mm}$ pedestal beam. Both were etched with Schaftmeister's reagent and illuminated using Diffractive Interference Contrast.

Measurements of the two tracks are given in Table 4.

The above-surface dimensions of the tracks are very similar. The pedestal track gave a diameter increase of just 9\% over the Gaussian track, with a corresponding reduction in height of $11 \%$, leading to a $18 \%$ increase in pitch circle diameter over the 3.5 Gaussian clad track. 
Table 4: Measurement data for $3.5 \mathrm{~mm}$ Gaussian and $1.25 \mathrm{~mm}$ pedestal HOE clad tracks

\begin{tabular}{|c|c|c|}
\hline & $\begin{array}{c}3.5 \mathrm{~mm} \\
\text { Gaussian clad } \\
\text { track }\end{array}$ & $\begin{array}{c}1.25 \mathrm{~mm} \\
\text { pedestal } \\
\text { HOE clad } \\
\text { track }\end{array}$ \\
\hline Width $(\mu \mathrm{m})$ & 1840 & 2000 \\
\hline $\begin{array}{c}\text { Above-surface } \\
\text { height }(\mu \mathrm{m})\end{array}$ & 572 & 510 \\
\hline $\begin{array}{c}\text { Penetration depth } \\
(\mu \mathrm{m})\end{array}$ & 376 & 140 \\
\hline Pitch circle diameter & 2130 & 2520 \\
\hline
\end{tabular}

The major difference between the clad tracks is in their dilution and mixing behaviour, shown by the differences in their penetration depths. Cladding with a pedestal HOE produces a clad track with a small penetration depth all along its width. In contrast, using a $3.5 \mathrm{~mm}$ Gaussian beam has no penetration at all at the edges, but has a large region of dilution in the centre of the track. This corresponds with experimental results found by Kim and Peng [3].

Since reflectivity calculations across the width of the track showed that the two beam shapes are absorbed in almost the same way, with the similarity in top-surface measurements bearing this out, it follows that the differences are in the longitudinal direction, in the way the two beams melt and cool the wire.

Both clad tracks were created using a traverse speed of $1.6 \mathrm{~mm} / \mathrm{s}$. Total heating time for the $3.5 \mathrm{~mm}$ Gaussian track was therefore approximately $2.2 \mathrm{~s}$; whereas for the $1.25 \mathrm{~mm}$ pedestal track, it was $0.8 \mathrm{~s}$. The pedestal track was heated, melted and cooled very quickly, whereas the Gaussian shape of the $3.5 \mathrm{~mm}$ beam track created an extended period of heating and cooling. This is shown by schematic in Figure 15.

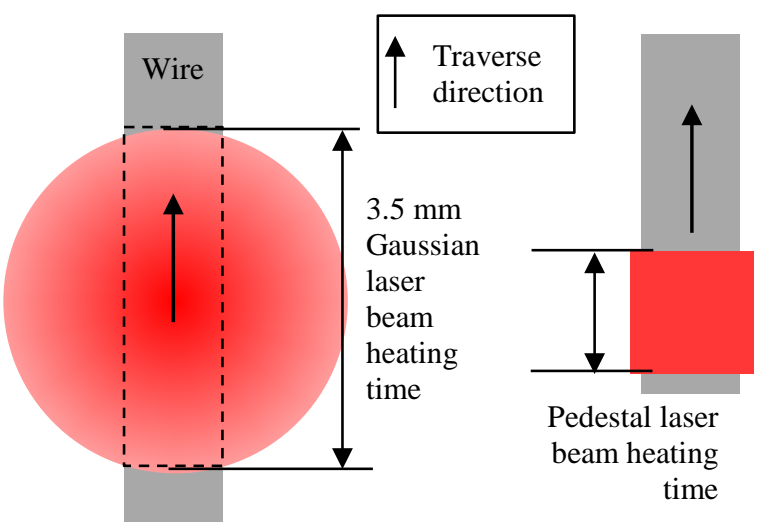

Figure 15: Schematic of thermal cycle times for $3.5 \mathrm{~mm}$ Gaussian beam vs. $1.25 \mathrm{~mm}$ pedestal beam
The longer thermal cycle undergone by the $3.5 \mathrm{~mm}$ Gaussian clad track means that the clad track is held in its molten state for longer, before solidifying. This allows more time for the marangoni flow in the track to create mixing with the substrate below; time that is not available for the pedestal track.

\section{Conclusions}

Heat conduction simulations gave realistic predictions of the results of the experiments. Melt pool formation and creation of clad tracks in experiments were in line with the predictions of modelling. These thermal models can now be further developed.

The top surface shape of the wire has a minor effect on the absorption from the laser beam. More heat is absorbed at the edges, where the angle of incidence is between 45 and $75^{\circ}$.

Using a flat beam profile reduces the amount of heat at the centre of the wire and inputs more at the edges. The square cross-section of the beam gives uniform heating time across the entire width of the wire, in contrast to the circular cross-section of the Gaussian beam, which causes the centre to be heated for twice as long as the edge. These result in superior melt pool formation characteristics.

Use of an enlarged $3.5 \mathrm{~mm}$ Gaussian beam makes the transverse absorption profile of the wire much more similar to that of a pedestal HOE beam. This allows the formation of a melt pool, although the extended heating time caused by the larger beam diameter causes a greater level of dilution compared to a pedestal HOE beam.

Although the $3.5 \mathrm{~mm}$ Gaussian beam gave equivalent absorption behaviour to a $1.25 \mathrm{~mm}$ pedestal diffractive beam across the width of the wire, the fact that it had to be so much larger to do so meant that the wire was heated for a longer period of time. This resulted in a large mixing zone concentrated in the centre of the track, which did not occur in the pedestal HOE track, which had a bottom surface that was largely flat.

Holographic Optics provide an improved way to control heat input into wire-fed laser cladding. Although using a larger Gaussian beam improves control compared to using a small beam, the benefits of mixing control by HOE's are superior.

Varying the aspect ratio of the beam footprint with a HOE means that they are capable of refinements that Gaussian beams are not; so a HOE beam could be made wider without being made longer with a separate 
pre and post heat treatment beam, which is not possible with a Gaussian beam.

\section{References}

[1] W. W. Duley, Laser Welding. John Wiley \& Sons Ltd., 1999.

[2] W. U. H. Syed, A. J. Pinkerton, and L. Li, “A comparative study of wire feeding and powder feeding in direct diode laser deposition for rapid prototyping,” Appl. Surf. Sci., vol. 247, no. 1-4, pp. 268-276, Jul. 2005.

[3] J.-D. Kim and Y. Peng, "Plunging method for Nd:YAG laser cladding with wire feeding," Opt. Lasers Eng., vol. 33, no. 4, pp. 299-309, Apr. 2000.

[4] E. Toyserkani, A. Khajepour, and S. Corbin, Laser Cladding. CRC Press LLC, 2005.

[5] R. Vilar, “Laser cladding,” J. Laser Appl., vol. 11, no. 2, p. 64, 1999.

[6] J. R. Tyrer and S. C. Noden, "Diffractive optical elements for manipulation of high power CO2 laser radiation - a feasibility study,” vol. 2789, 1996.

[7] J. Kell, J. R. Tyrer, R. L. Higginson, J. C. Jones, and S. Noden, "Laser weld pool management through diffractive holographic optics,” Mater. Sci. Technol., vol. 28, no. 3, 2011.

[8] R. L. Higginson, M. Gibson, J. Kell, and J. Tyrer, "Weld Pool Shaping and Microstructural Control Using Novel Computer Generated Holographic Optic Laser Welding of Steel and Stainless Steel," Mater. Sci. Forum, vol. 638-642, pp. 3673-3678, Jan. 2010.

[9] P. Bendeich, N. Alam, M. Brandt, D. Carr, K. Short, R. Blevins, C. Curfs, O. Kirstein, G. Atkinson, T. Holden, and R. Rogge, "Residual stress measurements in laser clad repaired low pressure turbine blades for the power industry,” Mater. Sci. Eng. A, vol. 437, no. 1, pp. 70-74, Nov. 2006.

[10] M. F. Modest, "Reflectivity and Absorptivity of Opaque Surfaces,” in LIA handbook of laser materials processing, 1st ed., J. F. Ready and D. F. Farson, pp. 175-181, Eds. 2001,

S. B. Boyden and Y. Zhang, "Temperature and Wavelength-Dependent Spectral Absorptivities of Metallic Materials in the Infrared.pdf,” $J$. Thermophys. Heat Transf., vol. 20, no. 1, 2006.

[12] E. Hecht, "Lasers and Laser Light," in Optics, 4th Editio., Pearson Education, Inc., pp. 594595, 2002.

\section{Meet the Authors}

Nicholas Goffin is a $\mathrm{PhD}$ research student at Loughborough University, conducting research into using holographic optics for wire-fed laser cladding. Shuai Hou is also a $\mathrm{PhD}$ research student, doing research in laser welding/cladding simulations. John Tyrer is Professor of Optical Instrumentation at Loughborough University and has been involved in a diverse range of laser processing research. Rebecca Higginson is a Senior Lecturer in Metallurgy in the Department of Materials at Loughborough University, specialising in microstructural development of ferrous and nom-ferrous alloys. 